\title{
A wind speed and fluctuation simulator for characterizing the wind lidar correlation method
}

\author{
S. Tomás ${ }^{\mathrm{a}}$, M. Sicard, J. Masjuan, M. N. Md. Reba, C. Muñoz and F. Rocadenbosch \\ Dept. of Signal Theory and Communications (TSC), Remote Sensing Lab. (RSLAB) \\ Universitat Politècnica de Catalunya (UPC) \\ Barcelona, Spain
}

\begin{abstract}
Aerosol distribution in the atmosphere is used as a wind-tracer by lidars since it is drifted by the wind and respond to its changes. Two methods are used: the correlation and the Doppler method. This first one is easier and cheaper to implement than the latter. It makes it competitive for retrieving wind speed profiles or estimating wind turbulence. However, its accuracy decreases significantly as the distance from the optical radiation source increases, hence the need to characterize the method by means of wind field profile simulations and validate it by comparing the retrieval of real wind velocities with that of cooperative instruments such as radiosoundings. In this paper, the bases of a 2D lidar signal simulator are presented. The relationship between wind fields and the aerosol concentration dynamics, and the way they relate to lidar signal returns is explained. The first results of the application of the correlation method for the retrieval of wind velocities from real data at the UPC are presented and compared to radiosoundings measurements.
\end{abstract}

Keywords: Correlation; wind retrieval; lidar; simulator; aerosol

\section{INTRODUCTION}

Profiles of tropospheric wind are a key data for validation of mesoscale meteorological forecasting models and climate monitoring. Wind profiling has manifold applications, as e.g. in environment studies for pollution monitoring and in aeronautic and defense business for characterizing aircraft turbulence or wind shear.

Wind lidar techniques are based either on the direct detection of the wind velocity or on the Doppler shift effect. The first technique uses methods of correlation between different lines of sight (LOS) using aerosols as wind tracers. Compared to Doppler systems it is simpler and faster to develop since it does not require a special reception system. One important feature is the ability to measure both radial and transverse winds.

In this work, the first attempts of the UPC Remote Sensing Lab. to retrieve wind fields are presented. In order to evaluate and design the most suitable correlation algorithm for the UPC lidar system, a lidar wind drifted signal simulator was developed.

\section{CORRELATION WIND LIDAR}

The existence of aerosol inhomogeneities is the basis for this method. They are spatial and temporal variations of aerosol microphysical characteristics such as concentration, size distribution and shape. Large scale (a few hundred meters) inhomogeneities in aerosol content are caused by an additional turbulent flux of particles which causes excitation of large scale instability in the vicinity of temperature inversion layers [1].

Lidar signals backscattered within the atmospheric boundary layer (ABL) are dominated by aerosol particles scattering. Fluctuations in aerosol content are easily detected with lidar. The observation of the drift of these spatial inhomogeneities can be used to determine wind velocities from the lidar signals.

Several temporal and/or spatial correlation techniques using lidar profiles of aerosol backscatter intensity have been developed. In wide scanning techniques, either a wide area or a volume section is covered. Narrow scanning procedures consist in a scanning through few LOS. Both spatial or temporal cross correlations are applied. In Buttler's procedure [2] the correlation algorithm followed an image pattern-matching procedure like the wide scanning techniques with time-height images (THI) for each LOS of a three-angle scan like the narrow scanning methods

\section{SIMULATOR OF 2D LIDAR SIGNALS}

The use of simulated signals to evaluate both the system performance and the processing algorithms is customary in many fields. Our goal is to apply physical concepts related to wind and aerosol dynamics to obtain THI images under controlled parameters to be applied in wind profiling algorithms.

\section{A. Inhomogeneities in the lidar equation}

The lidar signal is given by a time dependent single scattering lidar equation. We can split it in two terms, a static link budget term which includes the classic non fluctuating lidar equation which is a temporal averaged signal, and the dynamic link budget that is related to fluctuations in power given by aerosol inhomogeneities in the atmosphere. This second term is zero mean and time dependent.

$$
\begin{aligned}
P(R, t) & =\frac{K}{R^{2}}\left[\bar{\beta}(R)+\beta_{\text {aer }}^{\prime}(R, t)\right] T^{2}(R) \\
& =\frac{K}{R^{2}}\left[\bar{\beta}(R)+\frac{d \sigma_{a e r}}{d \Omega}(\pi) N^{\prime}{ }_{a e r}(R, t)\right] T^{2}(R)
\end{aligned}
$$

Where $K$ is the system constant, $R$ is the range, $T^{2}$ is the two-path transmission, $\bar{\beta}$ is the averaged backscatter

$\overline{{ }^{a} \text { Corresponding author: tomas.martinez@tsc.upc.edu }}$ 
coefficient, $\mathrm{d} \sigma(\pi) / \mathrm{d} \Omega$ is the differential cross section of the aerosol backscatter and $N^{\prime}$ aer is the zero mean fluctuating term of aerosol concentration.

Power fluctuations are generally considered proportional to the backscatter fluctuations avoiding the effect of an extinction fluctuating term. The backscatter fluctuating term $\beta$, ${ }_{\text {aer }}$ is considered proportional to the aerosol concentration field since large aerosols $(0.5$ to $3 \mu \mathrm{m})$ are mainly the responsible for those fluctuations [3]. The microphysical changes in aerosol content are related to the cross section and it is assumed constant for the observation time. Eventually the problem consists in simulating an evolution of $N_{\text {aer }}(R, t)$, a passive scalar field, according to wind parameters.

\section{B. Simulator strategies}

There are two strategies to simulate an advected aerosol concentration field. The more realistic splits the problem in the stochastic generation of a wind field and the application of a dynamic equation to obtain the scalar field. This equation is the advective-diffusive equation [4]. An alternative is to generate the passive scalar field based on several simplifications and limitations but linked however with wind field parameters. Independently of the approach chosen, a stochastic synthesis of turbulent scalar fields is needed. In this paper, the second approach under restricted conditions is considered.

A macroscopic description of concentration evolution in space and time is preferred over microphysical dynamics. In this work, physical relationships based on the turbulence theory are used to obtain the desired correlations in space and time altogether.

\section{Passive scalar field description}

Stochastic synthesis of turbulent fields is a well known topic in the wind engineering field and is based on generation of multivariate processes by a power spectral density matrix (PSD) [5]. Each matrix is representing a wind component. The strongest hypothesis in the simulator is the identification of the concentration field as a velocity component field. Such a simplification is given by the assumption of aerosol content as a conservative and passive (having no influence on wind) field. This implies that concentration fluctuations should replicate the spectral shape of wind components. This intuitive behaviour is present in the work of Kunkel [6].

According to Kolmogorov theory, the wind components spatial spectra have a $\mathrm{k}^{-5 / 3}$ dependence for a subinertial range,. Its counterpart in passive scalar fields has different expressions according to the subrange considered. For the inertialconvective subrange, the same $\mathrm{k}^{-5 / 3}$ dependence is given by the Obhukhov-Corsin spectrum [4]. Since aerosol concentration is a very slowly diffusing quantity, its fluctuations will have a viscous-convective range at small length scales (order of $\mathrm{cm}$ ) while the inertial convective regime is done at large scales (up to $10 \mathrm{~m}$ ).

The statistic homogeneity of the process for all the positions and ranges is a theoretical deficiency, as pointed out in [7]. This means that the covariance function only depends on the distance between two points. This is actually not true since wind velocity profiles are sheared and there is a position dependence. Nonetheless, some empirical results ensure that the spectral behaviour desired in the inertial-convective condition can be considered for separations of several hundreds of meters [6] or extended even to $1 \mathrm{~km}$ (here considering the structure function of the process) [3].

Simulations of pollutants concentration in plumes offer a more complete study considering not only the second order moments (cross spectra) but also the probability density function of the process [7]. The main difference with our case is that plumes have a located source. Atmospheric aerosol concentration statistics follow a lognormal law. At the moment, gaussianity of the processes will be assumed for simplicity.

Thus, the spectral descriptors employed for wind components fluctuations can be applied to have a passive scalar field synthesis with some additional considerations.

\section{Wind parameters and simulation regions}

Wind conditions are not directly related in the concentration simulated field because of the absence of a dynamic equation. Instead we can still relate the wind motion to the passive scalar field in several terms. The mean wind speed will be the conversion factor between temporal frequency and spatial frequency for the spectra expressions. The azimuth between mean wind direction and lidar LOS will determine a geometric transformation to obtain the final image. The mean wind field profile $U(z)$ has only vertical dependence (it is invariant in the horizontal plane). No geostrophic winds are considered. The profile is given by the logaritmic profile for the surface layer while it is extrapolated for the mixed layer as is done in [8].

The magnitude of wind fluctuation is described by its variance. This results in a variance of the lidar signal fluctuation which is due to both convective turbulence and vertical shear effect [4]. Hooper et al. [9] empirically modelled a signal variance from these two effects, which is not considered in this paper.

\section{E. Description of the simulator}

As stated above, the simulated field is a set of time series for an observation time where wind stationarity can be assumed (few minutes). The field is simulated according to the wind coordinates: $x$, longitudinal (horizontal and parallel to mean wind direction), $y$, lateral (horizontal, transversal to wind direction) and $z$, vertical. The main advantages are the knowledge of the wind components along these coordinates and the use of the Taylor's hypothesis which means that the $x$ dimension is interchangeable with time through the mean wind speed factor. The obtained pattern is considered "frozen" and can either represent an instantaneous space field or a temporal evolution of a set of space points spatially correlated as well.

The simplest case considered here is a set of time series for horizontal aligned points according to lateral coordinate $y$. The vertical coordinate $z$ is simply a constant. For the real lidar LOS's points, its slanted alignment require an additional term for $z$ shown in Eq. (4). 
The procedure to simulate a spatio-temporally correlated random field is based on Fourier synthesis and the PSD matrix $\mathbf{S}(f)=\left[S_{i j}(f)\right]$. Each component of the matrix $S_{i j}(f)$ is a crosspower spectrum evaluated at two different points and frequency-dependent. For a homogeneous turbulence field, the quadrature spectrum (the imaginary part) is relatively small compared to the cospectrum (real part). Thus, it is assumed that the cross-spectral density function is real. It is represented by:

$$
S_{i j}(f)=S\left(\vec{r}_{i}, \vec{r}_{j} ; f\right)=S_{i i}(f) S_{i j}(f) \gamma_{i j}^{2}(f)
$$

where $S_{i i}(f)$ and $\mathrm{S}_{j j}(f)$ are the power spectra of a scalar field evaluated for the first and second points, respectively (Eq. 3). The position vector, $\vec{r}_{i}=\left(y_{i}, z_{i}\right)$, follows the wind coordinate system and $x$ is not considered because of its exchangeability with time/frequency coordinate.

$$
S_{i i}(f)=S\left(\vec{r}_{i}, \vec{r}_{i} ; f\right)=S\left(z_{i}, f\right)=C_{0} \frac{A z_{i} / U\left(z_{i}\right)}{\left[1+B f z_{i} / U\left(z_{i}\right)\right]^{\frac{5}{3}}}
$$

$\mathrm{A}$ and $\mathrm{B}$ are coefficients empirically given depending on the magnitude considered. $U\left(z_{\mathrm{i}}\right)$ is the mean wind speed for the given height. We chose $\mathrm{A}=200$ and $\mathrm{B}=50$ for longitudinal wind velocity fluctuations like in [10]. $C_{0}$ is a normalization constant determined by integrating the spectra and making it equal to the desired variance, which is assumed on the order of unity.

The factor $\gamma_{i j}^{2}(f)$ is the coherence function relating the spatial correlation between two points. There are several models for such a function. We use a combination of the Schlez model for lateral separations [11] and the classic Davenport model for vertical separations:

$$
\gamma_{i j}^{2}(f)=\gamma^{2}\left(\vec{r}_{i}-\vec{r}_{j}, f\right)=\exp \left(-\frac{f}{U_{i j}} \sqrt{c_{y}^{2} \sigma^{2} \rho_{y}^{2}+c_{z}^{2} \rho_{z}^{2}}\right)
$$

where $U_{i j}$ is the mean velocity between $z_{i}$ and $z_{j}, \sigma^{2}$ is the field variance, $\rho_{y}$ and $\rho_{z}$ are the distances between points along $\mathrm{y}$ axis and $\mathrm{z}$ axis respectively. The coefficients $c_{y}$ and $c_{z}$ are empirically chosen as 3.6 and 6 , respectively, from [10].

The simulation consists in linearly transform a matrix $w_{k}(t)$ which represents time series of gaussian zero mean white noise for $k$ spatial points $(k=1$ to $\mathrm{M})$ into another one $n_{i}(t)$, correlating its components in space (Eq. 5). When applying the Fourier transform, this is done for each frequency and the temporal correlation is obtained. Each component $N_{i}(f)$ is the spectral representation of a temporal evolution for a position $\left(y_{i}, z_{i}\right)$. After that, an inverse Fourier transform allow the change of frequency to time (Eq. 6). Since gaussianity is kept in both linear transformations, gaussian processes are usually preferred in stochastic synthesis. As said previously, iterative corrections of the probability function are needed to reach lognormality. They are not considered here.

$$
\begin{aligned}
& N_{i}(f)=\mathbf{L}_{i k}(f) W_{k}(f) \\
& n_{i}(t)=\mathcal{F}^{-1}\left[N_{i}(f)\right]
\end{aligned}
$$

where $\mathbf{L}(f)$ is the lower triangular matrix from the Cholesky decomposition of the PSD matrix $\mathbf{S}(f)$.

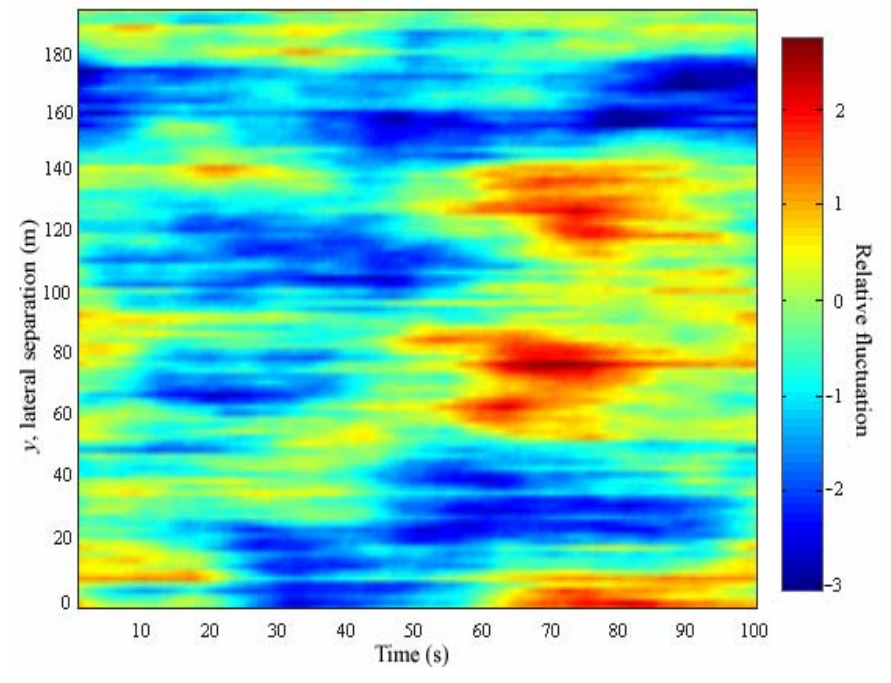

Figure 1. Simulated aerosol concentration (or equivalent backscatter) fluctuation field for a horizontal plane at $z=150 \mathrm{~m}, U(z)=10 \mathrm{~m} / \mathrm{s}$. Lateral range considered is $200 \mathrm{~m}$. The variance is close to unity. A slanted LOS with respect to the time (or $x$ ) axis gives the orientation of the lidar.

Finally, to transform the time series to lidar coordinates, a geometric transformation is done by interpolating the generated field along a crossed LOS (not shown above).

The study of the accuracy of the correlation method is assessed by splitting the several effects that are present in the fluctuations of the received power into independent contributions without losing likelihood. One can either study the influence of a particular effect or group them to make the performances more realistic.

\section{WIND FIELD RETRIEVAL FROM REAL DATA}

\section{A. UPC lidar system}

The UPC lidar system uses a 10-Hz pulsed Nd:YAG laser. Two elastic channels at 1064 and $532 \mathrm{~nm}$ are detected with avalanche photodiodes receivers. Scanning motions in elevation and azimuth are realized by stepping motors.

\section{B. Procedure}

The firsts measurements of wind advected aerosol signals have been done considering several parameters to obtain a suitable protocol. Following previous literature studies [2][6][9], scans around two LOS separated by angles of $0.5,1$ or $2^{\circ}$ are performed at a low elevation angle of $10^{\circ}$. The time resolution is $1 \mathrm{~s}$. (10 shots) and the observation time is less than $5 \mathrm{~min}$. The images used to estimate aerosol motions are slanted profiles of chronological power fluctuations (Fig.2). The maximum height is around $800 \mathrm{~m}$ which is the ABL height and corresponds to a signal-to-noise ratio less than unity.

A two-dimensional correlation for sub-images was perform to find the maxima. The imges' horizontal displacement is a time delay that gives an estimation of the transverse velocity when knowing the spatial separation between the two LOS (Fig.3). 

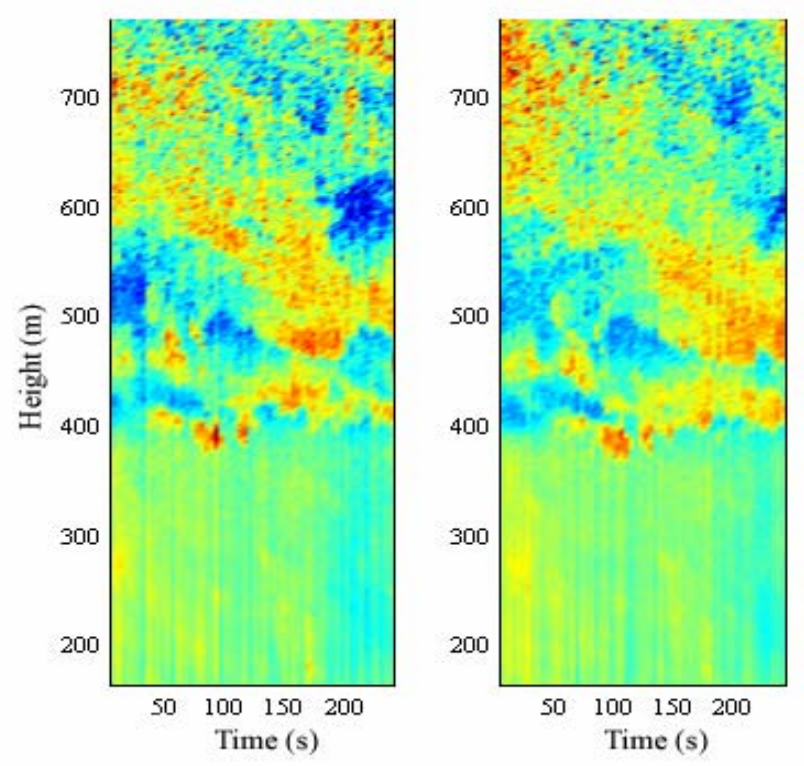

Figure 2. Zero mean, normalized fluctuation of power at two LOS. Observation time of $4 \mathrm{~min}$. Elevation angle $=10^{\circ}$ from ground. Azimuth step angle is $2^{\circ}$. Inclination on aerosol structures is an indicator of radial motion while differences in time coordinate gives an indication of transversal motion along base lines between the two LOS.
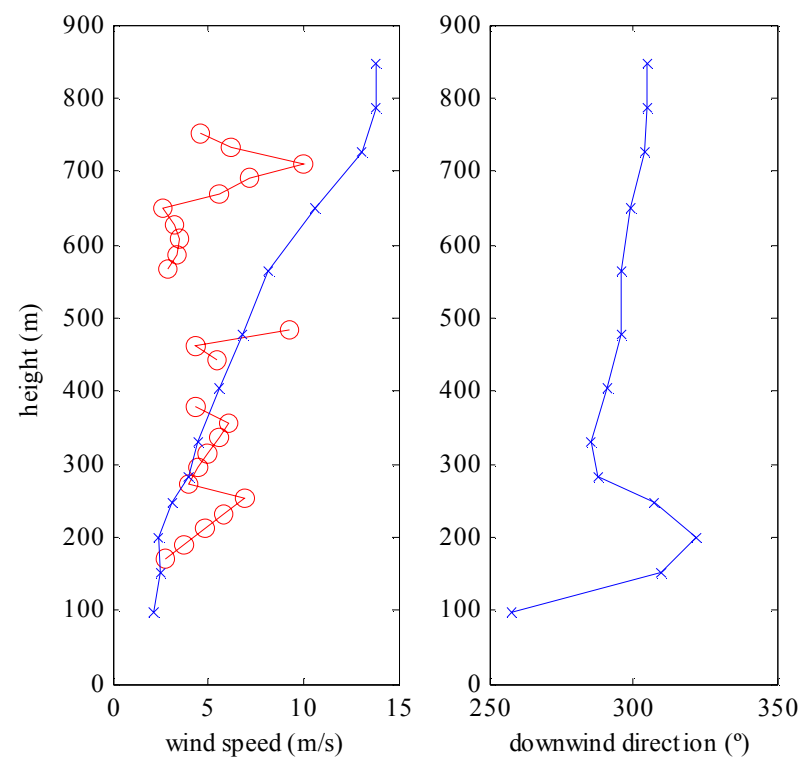

Figure 3. Wind speed and direction measurements from the UPClidar (1300UTC) and the radiosounding of the Servei Meteorologic de Catalunya (1200UTC) on 2007-2-23. (LEFT). Mean wind speed from the UPC lidar (circles, red) and the radiosoundings (crosses, blue); (RIGHT) downwind direction of radiosoundings. The lidar orientation is $225^{\circ}$.

The time difference between the radiosoundings and the lidar measurements can explain the difference observed in the wind speed profiles, most of all above $500 \mathrm{~m}$. We note that the wind speed variation with height (vertical shear) is the same for both instruments. The mean wind direction is $306^{\circ}$, orthogonal enough to the lidar orientation to consider almost a transversal motion.

The complete validation of the correlation method implies more lidar wind field retrievals together with radiosoundings and other cooperative instruments, and the evaluation of its performances using artificial, controlled profiles generated by the wind field and fluctuation simulator presented in this paper.

\section{ACKNOWLEDGMENT}

This work is supported by the European Union and FEDER funds under the EARLINET-ASOS project (EU Coordination Action, contract $\mathrm{n}^{\circ} 025991$ (RICA)), and the project titled "Technology development programme towards a European Extremely Large Telescope" (EU Specific Support Action, contract $\mathrm{n}^{\circ} 011863$ (RIDS)); by the MCYT (Spanish Ministry of Science and Technology) and FEDER funds under the projects TEC2006-07850/TCM and REN2003-09753-C02-02, the Complementary Actions CGL2006-26149-E/CLI and CTM2006-27154-E/TECNO, and the Special Action REN2002-12784-E; and the MITYC (Spanish Ministry of Industry, Tourism and Commerce) under the PROFIT project CIT-020400-2005-56. M. Sicard is grateful to the MCYT for the Ramón y Cajal position he holds. Md. Reba thanks the Local Government of Catalonia (Generalitat de Catalunya/ AGAUR) for his predoctoral fellowship.

\section{REFERENCES}

[1] T. Elperin, N. Kleeorin and I.Rogachevskii. "Mechanism of formation of aerosol and gaseous inhomogeneities in the turbulent atmosphere". Atmospheric Research 53, pp. 117-129, 2000.

[2] W. T. Buttler, C. Soriano, J M. Baldasano, and G. H. Nickel. "Remote sensing of three-dimensional winds with elastic lidar: explanation of maximum cross-correlation method". Boundary-Layer Meteorology, 97, pp. 305-328, 2001.

[3] M. J. Post. "Experimental measurements of atmospheric aerosol inhomogeneities". Optics Letters 6 (2), pp. 166-168, 1978.

[4] A. Celani, M. Cencini, M. Vergassola, E. Villermaux and D. Vincenzi. "Shear effects on passive scalar spectra". J. Fluid Mech. 523, pp. 99-108 2005.

[5] M. Di Paola. "Digital simulation of wind field velocity". J. of Wind Engng. and Industrial Aerodynamics, 74-76, pp. 91-109, 1998.

[6] K.E. Kunkel, E.W. Eloranta, and J.A. Weinman. "Remote determination of winds, turbulence spectra and energy dissipation rates in the boundary layer from lidar measurements". Journal of Aerosol Science, 37(5) pp. 979-985, 1980.

[7] M. Nielsen, P.C. Chatwin , H.E. Jorgensen, N. Mole, R.J. Munro and S. Ott. "Concentration fluctuations in gas releases by industrial accidents. Final Report”. Riso-R-1329(EN). Riso National Laboratory, Roskilde, Denmark, May 2002.

[8] G. Montero, R. Montenegro and J.M. Escobar. A 3-D diagnostic model for wind field adjustment. Journal of Wind Engineering and Industral Aerodynamics. 74-76, pp. 249-261, 1998.

[9] W. P. Hooper and E. W. Eloranta. "Lidar measurements of wind in the planetary boundary layer: the method, accuracy and results from joint measurements with radiosonde and kytoon". J. Climate Appl. Meteor., 25, pp. 990-1001, 1986.

[10] M. Shinya and A. Fournier. "Stochastic motion - motion under the influence of wind”. EUROGRAPHICS'92 Proceedings, 1992.

[11] W. Schlez and D. Infield. "Horizontal, two point coherence for separations greater than measurement height". Boundary-Layer Meteorology. 87, pp. 459-480, 1998. 